\title{
Wenn Wenige den Ausschlag geben ... Strategisches Erststimmenwählen bei deutschen Bundestagswahlen 1994-2009
}

\author{
Michael Herrmann
}

Zusammenfassung: Anhand der offiziellen Wahlergebnisse aus Wahlkreisen in Ostdeutschland wird in diesem Beitrag untersucht, welche Bedeutung strategisches Erststimmenwählen bei deutschen Bundestagswahlen hat. Die Analyse zeigt: 1) Es wurde tatsächlich strategisch gewählt; 2) je nach Bundestagswahl profitierten CDU oder SPD am häufigsten von strategischem Wählen; 3) bis zu 9\% der Wähler im Wahlkreis wählten strategisch; 4) diese Zahl war groß genug, um in einer Reihe von (überhangmandatsrelevanten) Wahlkreisen den Wahlausgang und damit die Zusammensetzung des Bundestages zu beeinflussen.

Schlüsselwörter: Strategisches Wählen · Bundestagswahl · Erststimme · Wahlkreis · Mehrheitswahl

\section{When a Few Tip the Scales ... Strategic Voting in German Constituencies 1994-2009}

\begin{abstract}
Based on official election records from East German constituencies, this paper examines the importance of strategic voting in German Bundestag elections. The results show that: (1) voters do indeed cast strategic ballots; (2) overall, the CDU and SPD benefit most often from strategic votes; (3) up to $9 \%$ of voters in the constituency cast strategic votes; and (4) that number was large enough to tip the scales in a number of constituencies, thus affecting the allocation of surplus seats in the Bundestag.
\end{abstract}

Keywords: Strategic voting · German federal election · Constituency $\cdot$ Single member district . Plurality election

Dipl.-Soz. M. Herrmann (两)

Fachbereich Politik- und Verwaltungswissenschaft, Universität Konstanz,

Universitätsstraße 10, Postfach 92, 78457 Konstanz, Deutschland

E-Mail: michael.herrmann@uni-konstanz.de 


\section{Einleitung}

In einer einfachen Mehrheitswahl steht ein Wähler vor der Entscheidung, seine Stimme einer anderen Partei als der bevorzugten zu geben, sofern diese keine Chance hat, den Sieg davon zu tragen. Dieses sogenannte strategische Wählen zielt darauf ab, einen möglichst günstigen Wahlausgang herbeizuführen - im Extremfall könnte ein einzelner strategischer Wähler den Ausschlag dafür geben, welche Partei die Wahl gewinnt. Aufgrund seiner entscheidenden Bedeutung für den Ausgang politischer Abstimmungen erfreut sich das Phänomen des strategischen Wählens seit jeher eines großen Interesses in der Fachwelt. Erstaunlicherweise wissen wir aber bis heute kaum etwas darüber, ob strategisches Wählen tatsächlich den Ausgang von Massenwahlen beeinflusst. Zwar ist inzwischen durch zahlreiche Studien nachgewiesen, dass Wähler unter den Bedingungen einfacher Mehrheitswahl strategische Verhaltensmuster zeigen (z. B. Heath et al. 1991; Cox 1997; Blais u. Nadeau 1996; Alvarez u. Nagler 2000; Karp et al. 2002; Fisher 2004; Gschwend 2007; Herrmann u. Pappi 2008). Die Frage nach der politischen Relevanz strategischen Wählens ist dagegen weitgehend ungeklärt.

Macht strategisches Wählen einen Unterschied? Empirische Studien können diese Frage bisher nicht beantworten, da sich mit bestehenden Ansätzen kaum verlässliche Hochrechnungen über das Ausmaß strategischen Wählens innerhalb von Wahlkreisen vornehmen lassen. In diesem Beitrag wird ein Modell entwickelt, das es erlaubt, solche Hochrechnungen anzustellen. Angewandt auf ostdeutsche Wahlkreise bei den Bundestagswahlen 1994-2009 liefert das Modell Aufschluss darüber, wie empfänglich Anhänger von CDU, SPD und PDS/DIE LINKE für strategische Anreize in ihrem Wahlkreis sind, welche Parteien am meisten von strategischen Stimmen profitierten bzw. welchen Parteien strategisches Wählen am meisten schadete, wie viel Prozent der im Wahlkreis abgegebenen Stimmen strategischer Natur waren und wie sich strategisches Wählen auf die Sitzverteilung im Bundestag auswirken kann. Insgesamt zeigen die Ergebnisse, dass strategisches Erststimmenwählen tatsächlich politische Relevanz entfalten kann.

Was mit strategischem Erststimmenwählen genau gemeint ist und welche Rolle es bei deutschen Bundestagswahlen (insbesondere in ostdeutschen Wahlkreisen) spielt, ist Gegenstand des folgenden Abschnitts. Im Anschluss daran wird aufgezeigt, warum bisherige Studien keine Antwort auf die Frage nach der Bedeutsamkeit strategischen Wählens geben konnten, bevor schließlich das Modell und die Ergebnisse der Schätzung strategischen Erststimmenwählens vorgestellt und diskutiert werden. Es sei vorab darauf hingewiesen, dass sich dieser Beitrag ausschließlich mit dem Wahlverhalten bezüglich der Erststimme beschäftigt. Die Wahlentscheidung mit der Zweitstimme ist nicht Gegenstand des Interesses. Zwar ist auch das Zweitstimmenwählen generell nicht frei von Anreizen zur strategischen Stimmabgabe (etwa zur Beeinflussung der Koalitionsbildung), im Folgenden soll die Bezeichnung, strategisches Wählen` jedoch synonym mit ,strategischem Erststimmenwählen' gebraucht werden.

\section{Strategisches Erststimmenwählen und seine politischen Konsequenzen}

Am 15. November 1994 wurde Helmut Kohl mit 338 von 337 erforderlichen Stimmen in seine vierte und letzte Amtsperiode als Bundeskanzler gewählt. Unter den CDU-Abge- 
ordneten, die für ihn stimmten, befanden sich insgesamt zwölf Abgeordnete, die mit sogenannten Überhangmandaten in den Bundestag eingezogen waren; die meisten davon aus den neuen Bundesländern. Überhangmandate entstehen, wenn eine Partei in einem Bundesland mehr Mandate in Wahlkreisen gewinnt, als ihr aufgrund ihrer landesweit errungenen Zweitstimmen zustehen. Das heißt, Überhangmandate erlauben es einer Partei, ihre Sitzstärke über ihren reinen Zweitstimmenanteil hinaus zu vergrößern. Die CDU konnte auf diese Weise allein in Mecklenburg-Vorpommern, Sachsen, Sachsen-Anhalt und Thüringen insgesamt zehn zusätzliche Mandate gewinnen. Die SPD kam im Vergleich dazu nur in Brandenburg zu insgesamt drei Überhangmandaten.

Was wäre passiert, wenn die SPD der CDU in Sachsen oder Thüringen den einen oder anderen Wahlkreis abgerungen hätte? Für die CDU hätte dies einen Verlust von Überhangmandaten bedeutet und damit eine Verringerung ihrer Sitzstärke im Bundestag. Darüber hinaus hätte die SPD je nach Zweitstimmenlage in den einzelnen Ländern durch Überhangmandate zusätzliche Sitze gewinnen können. Für die Wiederwahl Helmut Kohls wäre dies wohl nicht ohne Folgen geblieben. Nun war es aber nicht so, möchte man einwenden, auch wenn der Ausgang knapp war. Letztlich hatte die CDU in den betreffenden Wahlkreisen eben doch mehr Anhänger als die SPD. Aber stimmt das? Hatte die CDU wirklich mehr Anhänger als die SPD? Oder könnte es nicht sein, dass Anhänger der PDS als drittstärkste Partei sich entschlossen haben, in das Rennen um den Ausgang im Wahlkreis einzugreifen, d.h. ihre Stimme derjenigen Partei zu geben, die ihrer Meinung nach eher den Sieg davon tragen sollte?

Einfache Mehrheitswahlen sind wie geschaffen, um durch strategisches Verhalten seitens der Wähler ,manipuliert` zu werden. Im Gegensatz zur Verhältniswahl zählt bei einfacher Mehrheitswahl nur, welcher Kandidat die meisten Stimmen auf sich vereinigt; einen Preis für den zweiten Platz gibt es nicht. Das hat den Effekt, dass nur eine Stimme für einen der beiden aussichtsreichsten Kandidaten über den Wahlausgang entscheiden kann. Die Chance, mit seiner Stimme einem anderen Kandidaten zum Sieg zu verhelfen, ist im Vergleich dazu unbedeutend. Anders ausgedrückt: Jede Stimme für einen dritt- oder viertplatzierten Kandidaten ist ,vergeudet'. Sofern der eigene Wunschkandidat also nicht zu den beiden führenden Bewerbern gehört, liegt es nahe seine Stimme einzusetzen, um dem ,kleineren Übel' unter den beiden aussichtsreichen Bewerbern zum Sieg zu verhelfen.

Als Beispiel stelle man sich einen Wahlkreis vor, in dem $40 \%$ der Wähler hinter dem Amtsinhaber stehen. Der Amtsinhaber sieht sich zwei Herausforderern A und B mit jeweils 38 und 22\% Anhängerschaft im Wahlkreis gegenüber. Sofern jeder Wähler für seinen bevorzugten Kandidaten stimmt, wird der Amtsinhaber wiedergewählt. Entscheidet sich aber nur ein kleiner Teil der Anhänger von B, die Herausforderer A dem Amtsinhaber vorziehen, für A zu stimmen, so verliert der Amtsinhaber die Wahl. Unter einfacher Mehrheitswahl muss daher nicht unbedingt der Kandidat oder die Partei mit den meisten Anhängern gewinnen, sondern auch jener, welcher die meisten strategischen Stimmen von Anhängern schlechter platzierter Kandidaten auf sich vereinigt. ' Solange mehr als zwei Bewerber zur Wahl stehen, besitzen Anhänger des drittplatzierten oder schlechter platzierter Kandidaten stets einen Anreiz, ihre Stimme einem der beiden führenden

1 Das muss sich nicht zwangsläufig zum Nachteil des führenden Kandidaten auswirken. Im obigen Beispiel könnte man auch davon ausgehen, dass es Anhänger von B gibt, die den Amtsinhaber dem Herausforderer A vorziehen und deswegen strategisch für den Amtsinhaber stimmen (um sicherzustellen, dass jener wiedergewählt wird). 
Kandidaten zu geben. ${ }^{2}$ Intuitiv gesprochen entspricht diese Entscheidung der Wahl eines zweitbesten Kandidaten, um ,das Schlimmste zu verhindern'.

Für die Wiederwahl Helmut Kohls sind die Implikationen strategischen Wählens klar: Bereits eine geringe Zahl von PDS-Anhängern hätte in einem Wahlkreis darüber entscheiden können, ob der Kandidat der CDU oder der SPD den Sieg davon trägt. In Verbindung mit der Überhangmandatsregelung ist es also durchaus vorstellbar, dass Helmut Kohl seine letzte Kanzlermehrheit dem strategischen Wählen von PDS-Anhängern in Ostdeutschland zu verdanken hatte. Ob der Ausgang der Wahl tatsächlich verändert wurde, hängt letztlich davon ab, wie viele strategische Wähler im Wahlkreis sich für den Favoriten bzw. den Zweitplatzierten entschieden haben. Das zu bestimmen, ist eine empirische Aufgabe, der wir im Folgenden nachgehen wollen. Das Augenmerk der Analyse liegt dabei nicht nur auf den Wahlkreisen bei der Bundestagswahl 1994 - der Wiederwahl Kohls -, sondern auf ostdeutschen Wahlkreisen insgesamt.

Für die Untersuchung der politischen Relevanz strategischen Wählens liegt es nahe, ostdeutsche Wahlkreise zu betrachten, sowohl aus inhaltlichen als auch aus technischen Gründen. Rein technisch gesehen, geht das in diesem Aufsatz entwickelte Modell von einem Dreiparteienwettbewerb aus, was eine Anwendung auf westdeutsche Wahlkreise ausschließt (mehr dazu in den folgenden Abschnitten). Inhaltlich spricht dafür, dass anders als im Westen in den neuen Bundesländern bei jeder Bundestagswahl bisher zahlreiche Überhangmandate entstanden sind: Der Median liegt bei 7,5 Mandaten pro Bundestagswahl - in den alten Bundesländern dagegen nur bei zwei -, und mit Ausnahme von Berlin traten bisher in jedem ostdeutschen Bundesland bei zwei oder mehr Bundestagswahlen Überhangmandate auf. ${ }^{3}$ Zwar fielen bei der Bundestagswahl 2009 viele Überhangmandate im Westen an (erstmals mehr als im Osten), trotzdem kamen auch hier wieder acht Mandate aus den neuen Bundesländern. In den vergangenen Wahlen kam den Ergebnissen in ostdeutschen Wahlkreisen damit jedes Mal eine unmittelbare Relevanz für die Sitzverteilung im Bundestag zu. Wenn sich strategisches Erststimmenwählen also als politisch bedeutsam erweisen sollte, dann am ehesten in diesem Teil Deutschlands.

\section{Schwierigkeiten bei der Bestimmung des Ausmaßes strategischen Wählens}

Ungeachtet der immensen Zahl an Studien zu strategischem Wählen, insbesondere in der angelsächsischen Literatur, ist es bisher kaum gelungen, präzise Schätzungen über das Ausmaß strategischen Wählens innerhalb von Wahlkreisen abzugeben. Ebenso wenig wissen wir darüber, wie groß die Zahl strategischer Stimmentransfers zwischen Parteien in einzelnen Wahlkreisen ausfällt.

2 In Wahlen mit nur zwei Bewerbern besteht keine Möglichkeit, strategisch einen zweitbesten Kandidaten zu wählen, um das Schlimmste zu verhindern. Jeder Wähler sollte deshalb für seinen favorisierten Kandidaten stimmen.

3 Zur Entstehung von Überhangmandaten, ihrer politischen Relevanz und ihrem verfassungsrechtlichen Stellenwert siehe Behnke (2003, 2007). Die Anzahl von Überhangmandaten getrennt nach Ost und West (in Klammern) beträgt 1990: 6 (0), 1994: 13 (3), 1998: 4 (1), 2002: 4 (1), 2005: 11 (5) und 2009: 8 (17). 
Ein Grund für diesen Mangel an Ergebnissen liegt darin begründet, dass die überwiegende Mehrzahl von Studien strategisches Wählen auf der Basis von Umfragedaten untersucht (z. B. Black 1978; Cain 1978; Niemi et al. 1992; Blais u. Nadeau 1996; Ordeshook u. Zeng 1997; Fisher 2004; Gschwend 2007). Umfragedaten sind zwar einerseits unentbehrlich, um strategisches Wählen nachzuweisen, sie eignen sich andererseits aber schwerlich zur Untersuchung von Wählerwanderungen innerhalb von Wahlkreisen. Im Prinzip müsste dafür in jedem Wahlkreis eine repräsentative Stichprobe von Wahlberechtigten befragt werden. Bisher gibt es keine Studie, die das auch nur annähernd leisten könnte. Mit herkömmlichen, national repräsentativen Umfragen lassen sich lediglich Schätzungen über das nationale Ausmaß strategischen Wählens abgeben (z. B. Blais et al. 2001; Blais et al. 2005; Alvarez u. Nagler 2000; Alvarez et al. 2006). Beispielsweise schätzen Herrmann und Pappi (2008), dass bei den Bundestagswahlen 1998 und 2002 etwa 3\% der Wähler strategisch ihre Erststimme einer anderen Partei gaben. Solche Schätzungen maskieren aber den Effekt, den strategisches Wählen in einzelnen Wahlkreisen haben kann. Schließlich bestehen nicht in jedem Wahlkreis dieselben Anreize, strategisch zu wählen, und auch der Wahlausgang kann unterschiedlich knapp ausfallen. Außerdem ist es theoretisch wie politisch unbedeutend, wie viel Prozent der Wähler bundesweit strategisch gewählt haben, da sich strategisches Wählen auf die Beeinflussung des Ergebnisses im Wahlkreis richtet. Eine über alle Wahlkreise hinweg geschätzte Größe kann hier keinen Aufschluss geben.

Eine alternative Vorgehensweise besteht darin, strategisches Wählen auf der Basis von Aggregatdaten zu untersuchen, das heißt, aus den tatsächlichen Wahlergebnissen im Wahlkreis auf das Ausmaß strategischen Wählens zu schließen (vgl. Galbraith u. Rae 1989; Johnston u. Pattie 1991; Fieldhouse et al. 1996). Damit lässt sich zwar im Prinzip das Ausmaß strategischen Wählens auf Wahlkreisebene studieren. Da im beobachteten Wahlkreisergebnis strategische Stimmen aber bereits enthalten sind, benötigt man zusätzliche Informationen darüber, wie die Anhängerschaften der einzelnen Parteien im Wahlkreis verteilt sind. Verlässliche Daten über die Parteipräferenzen der Wähler im Wahlkreis sind mangels repräsentativer Umfragen auf Wahlkreisebene in der Regel aber nicht verfügbar, und vergangene Wahlergebnisse als Maßstab zu nehmen, verlagert eher das Problem, statt es zu lösen.

Im Gegensatz zu reinen Mehrheitswahlsystemen bietet das deutsche Mischwahlsystem (mit seiner Kombination von einfacher Mehrheitswahl und Verhältniswahl) die Möglichkeit, strategisches Wählen mithilfe eines Vergleichs von Erst- und Zweitstimmenergebnis auf Wahlkreisebene zu untersuchen. Einige Studien machen sich dieses Potenzial zu Nutze: Cox (1997) und Bawn (1999) etwa sowie auch Gschwend et al. (2003) betrachten jeweils die Differenz zwischen dem Zweit- und Erststimmenergebnis kleiner Parteien (FDP und Grüne) und können zeigen, dass diese mit der Knappheit des Erststimmenergebnisses der beiden großen Parteien (CDU und SPD) im Wahlkreis ansteigt. Zwar ist dies ein deutlicher Hinweis auf strategisches Wählen, die Frage nach der Größenordnung und politischen Relevanz des Phänomens bleibt damit aber weiterhin unbeantwortet. Erstens spielt aus theoretischer Sicht für strategisches Wählen die Knappheit des Wahlergebnisses nur eine untergeordnete Rolle. Viel wichtiger aus der Sicht des Wählers ist, wie gering die Chancen des bevorzugten Kandidaten sind, den Sieg davon zu tragen, und das hängt nur indirekt von der Knappheit des Rennens zwischen den beiden führenden Kandidaten ab (vgl. Myatt 2007). Zweitens spiegelt die Knappheit des Wahlausgangs erneut den Zustand wider, nachdem bereits strategisch gewählt wurde. Die Knappheit 
wäre also vielmehr das Produkt als die Voraussetzung für strategisches Wählen. Und drittens betrachten die Autoren lediglich die Stimmenverluste kleiner Parteien. Damit bleibt die Frage offen, welcher Partei sich strategische Wähler im Wahlkreis zugewandt haben; mit anderen Worten: Strategische Wählerwanderungen auf Wahlkreisebene lassen sich mit bisherigen Ansätzen nicht nachvollziehen.

Im Folgenden wird daher aufbauend auf einem entscheidungstheoretischen Modell strategischen Wählens ein statistisches Modell entwickelt, das es erlaubt, unter gleichzeitiger Berücksichtigung von Stimmenverlusten und -gewinnen von Parteien die strategischen Wählerwanderungen im Wahlkreis nachzuvollziehen. Um die Umsetzbarkeit des Modells zu gewährleisten, wird bei der Modellierung von einem Dreiparteienwettbewerb ausgegangen. Diese Annahme erscheint in Wahlkreisen in Ostdeutschland (ohne West-Berlin) generell gerechtfertigt. CDU, SPD und PDS (ab 2007: DIE LINKE) errangen dort stets den Löwenanteil (ca. 90\%) der Erststimmen. ${ }^{4}$ Dabei gewann die drittplatzierte Partei im Wahlkreis (meist die PDS) mit durchschnittlich 20\% der Stimmen stets mit deutlichem Abstand vor den viert- und fünftplatzierten Parteien mit jeweils 4 bis $6 \%$ der Stimmen, was die Annahme eines Dreiparteienwettbewerbs insgesamt plausibel erscheinen lässt. Eine Ausnahme stellt lediglich die Bundestagswahl 1990 - die erste nach der Wiedervereinigung - dar. Diese Wahl wird im Folgenden nicht berücksichtigt, da hier FDP und PDS in vielen Wahlkreisen etwa gleich stark waren und sich zusammen mit CDU und SPD die vorderen Plätze im Wahlkreis teilten. Die Annahme eines Dreiparteienwettbewerbs auf Wahlkreisebene erscheint vor diesem Hintergrund fraglich. Zudem sollte davon ausgegangen werden, dass die Wähler in der ersten Wahl nach der Wiedervereinigung die politischen Konsequenzen des für sie neuen Wahlsystems noch nicht ausreichend antizipieren konnten, um strategisch zu wählen (vgl. Lijphart 1994, S. 88; Reed 1990, S. 342). Der Fokus der Analyse liegt daher auf dem Zeitraum 1994 bis 2009.

\section{Die Modellierung strategischer Wählerwanderungen}

\subsection{Die Entscheidung des Wählers}

Bei einfacher Mehrheitswahl mit drei Parteien lässt sich der entscheidungstheoretische Kalkül eines Wählers folgendermaßen beschreiben: Wähle die bevorzugte Alternative, sofern der erwartete Nutzen dieser Handlung größer ausfällt als der erwartete Nutzen der Wahl der zweitbesten Alternative. ${ }^{5}$ Formal lässt sich diese Regel in der bekannten Ungleichung ausdrücken (vgl. McKelvey u. Ordeshook 1972; Black 1978; Hoffman 1982):

$$
p_{12}\left(u_{1}-u_{2}\right)+p_{13}\left(u_{1}-u_{3}\right) \geq p_{12}\left(u_{2}-u_{1}\right)+p_{23}\left(u_{2}-u_{3}\right) \text {. }
$$

Die einzelnen $u$-Terme in Ungleichung (1) stehen je nach Subskript für den Nutzen eines Sieges der erst-, zweit- oder drittpräferierten Partei, wobei $u_{1} \geq u_{2} \geq u_{3}$. Die $p$-Terme

4 Zum Vergleich: In Großbritannien kamen die drei großen Parteien Konservative, Labour und Liberale in den vergangenen vier Parlamentswahlen ebenfalls auf jeweils $90 \%$ der Stimmen (Rallings u. Thrasher 2007; http://news.bbc.co.uk/2/shared/election2010/results/. Zugegriffen: 10. August 2010).

5 Die Wahl der drittbesten Alternative kann nicht optimal sein, daher zählt nur der Vergleich der beiden besseren Optionen. 
bezeichnen die Wahrscheinlichkeit, dass die eigene Stimme den Wahlausgang zwischen der erst- und zweit-, erst- und dritt- oder zweit- und drittpräferierten Partei entscheidet. Diese sogenannten Pivot-Wahrscheinlichkeiten geben an, wie wahrscheinlich es ist, dass zwei Parteien genau gleich viele Stimmen erhalten, sodass die Stimme des Wählers den Ausschlag geben würde. ${ }^{6}$ Natürlich ist es in Massenwahlen sehr unwahrscheinlich, dass es zu einem derartigen Ereignis kommt, aber für die Wahlentscheidung spielt die absolute Wahrscheinlichkeit keine Rolle, sondern vielmehr die relative Wahrscheinlichkeit, also die Frage, wie viel wahrscheinlicher ein Ereignis gegenüber einem anderen ist. Auf diesen Punkt werden wir später noch genauer eingehen. Zunächst finden wir in Ungleichung (1) das Kriterium zu strategischem Wählen. Ein Wähler entscheidet sich für die aus seiner Sicht zweitbeste Alternative, wenn die rechte Seite der Ungleichung größer ist als die Linke, andernfalls wählt er die von ihm bevorzugte Partei.

Auf der linken Seite der Ungleichung finden wir den Erwartungsnutzen einer Wahl der bevorzugten Partei. Dieser Nutzen kann auf zweierlei Art realisiert werden: zum einem indem man mit der eigenen Stimme der bevorzugten Partei zum Sieg über die zweitpräferierte Partei verhilft. Gegenüber dem Wahlsieg der zweitpräferierten Partei stiftet dies dem Wähler einen zusätzlichen Nutzen von $u_{1}-u_{2}$. Gewichtet wird der Nutzen mit der Wahrscheinlichkeit, dass der Wähler mit seiner Stimmabgabe dieses Ergebnis herbeiführt. Die zweite Art, auf die eine Stimmabgabe für die bevorzugte Partei nützlich sein könnte, ist jene, der bevorzugten Partei zum Sieg über die am wenigsten gewünschte Partei zu verhelfen. In diesem Fall würde der Wähler mit Wahrscheinlichkeit $p_{13}$ einen Nutzengewinn von $u_{1}-u_{3}$ verbuchen.

Analog dazu steht auf der rechten Seite der Erwartungsnutzen einer Stimmabgabe zugunsten der zweitpräferierten Partei. Dieser ergibt sich wiederum aus zwei Szenarien: Entweder verhilft die eigene Stimme der zweitpräferierten Partei zum Sieg über die bevorzugte Partei. Dies ist dasselbe Szenario wie im vorherigen Abschnitt, nur mit dem Unterschied, dass der Wähler der ,falschen' Partei zum Sieg verhelfen würde und so mit Wahrscheinlichkeit $p_{12}$ einen Verlust von $u_{2}-u_{1}$ hinnehmen müsste. Die einzige Möglichkeit für den Wähler, seinen Nutzen mit der Wahl der zweitpräferierten Partei zu steigern, besteht darin, dass jener der zweitpräferierten Partei zum Sieg über die am wenigsten gewünschte Partei verhilft. In diesem Fall hätte der Wähler mit Wahrscheinlichkeit $p_{23}$ einen Nutzengewinn von $u_{2}-u_{3}$.

Man sieht bereits an dieser Stelle, dass sich die Wahl der zweitbesten anstelle der bevorzugten Partei nur lohnen kann, wenn die Wahrscheinlichkeiten $p_{12}$ und $p_{13}$ wesentlich kleiner sind als $p_{23}$, d.h., wenn die Wahrscheinlichkeiten eines Patts zwischen der favorisierten Partei und jeder der beiden anderen Parteien kleiner sind als die Wahrscheinlichkeit eines Patts zwischen der zweitpräferierten und der ungeliebten Partei. Dieser Anreiz vergrößert sich zudem, je schlechter ein Wahlsieg der ungeliebten gegenüber der zweitbesten Partei bewertet wird, d.h. je größer $u_{2}-u_{3}$. Dieser Zusammenhang wird noch klarer, wenn wir Ungleichung (1) wie folgt umformen (Herleitung im Anhang):

$$
\frac{u_{1}-u_{3}}{u_{2}-u_{3}} \geq \frac{2 p_{12}+p_{23}}{2 p_{12}+p_{13}} \text {. }
$$

6 Im Einklang mit der Literatur wird ein Dreiparteienpatt ausgeschlossen (vgl. etwa Myerson u. Weber 1993). 
Ungleichung (2) besagt, dass ein Wähler seine bevorzugte Partei wählen sollte, solange dessen Präferenz für diese Partei gegenüber der zweitpräferierten Alternative größer ist als die Chance eines Patts zwischen der zweit- und drittpräferierten Partei gegenüber eines Patts zwischen der favorisierten Partei und der drittpräferierten Partei (vgl. Hoffman 1982; Palfrey 1989; Fey 1997). Diese Darstellung des Wahlkriteriums ist äquivalent zur Darstellung in Ungleichung (1), jedoch wesentlich intuitiver.

Betrachten wir einen Wähler, dessen Nutzen von einem Sieg der drittpräferierten Partei, $u_{3}$, null ist. Dieser Wähler müsste sich lediglich fragen: Sofern es zu einem Patt kommen sollte, wie steht die Chance eines Patts zwischen meiner dritt- und zweitliebsten Partei gegenüber einem Patt zwischen meiner drittliebsten und bevorzugten Partei? Nehmen wir an, der Wähler käme zu dem Schluss, dass die Chance 2:1 beträgt - das erste Patt, $p_{23}$, ihm also doppelt so wahrscheinlich erschiene wie das zweite, $p_{13}-$, so würde er, grob gesprochen, seine bevorzugte Partei nur wählen, wenn ihm deren Sieg mindestens doppelt so wichtig wäre wie ein Sieg der zweitliebsten Partei. Bedeutet ihr Sieg ihm weniger, so würde er sich strategisch für die Wahl seiner zweitliebsten Partei entscheiden. Streng genommen ist diese Aussage nicht vollständig korrekt, da sie die Wahrscheinlichkeit eines Patts zwischen der favorisierten und der zweitpräferierten Partei, $p_{12}$, ausblendet bzw. unterstellt, diese Wahrscheinlichkeit sei null (generell gilt: je größer $p_{12}$, desto schwächer wird die oben beschriebene Tendenz). Inhaltlich ändert sich dadurch jedoch nichts. Für strategisches Wählen spielt letztlich nur das Verhältnis von $p_{23} \mathrm{zu} p_{13}$ eine Rolle. Je größer $p_{23}$ gegenüber $p_{13}$, desto eher sollte ceteris paribus strategisch gewählt werden.

Die Darstellung in Ungleichung (2) zeigt zwei wichtige Eigenschaften strategischen Wählens auf: Erstens kommt es bei der Wahlentscheidung nicht darauf an, wie wahrscheinlich es ist, dass eine Pattsituation (egal welche) entsteht. In Massenwahlen sind solche Wahrscheinlichkeiten extrem gering. Worauf es ankommt, ist der Vergleich der Wahrscheinlichkeiten, und der funktioniert auch mit extrem kleinen Wahrscheinlichkeiten: Ein Ereignis mit Wahrscheinlichkeit 0,00002 ist immer noch doppelt so wahrscheinlich wie ein Ereignis mit Wahrscheinlichkeit 0,00001. Um strategisch zu wählen, muss der Wähler die absoluten Wahrscheinlichkeiten noch nicht einmal kennen, sondern lediglich deren Verhältnis zueinander abschätzen können. ${ }^{7}$

Zweitens kann man aus Ungleichung (2) ablesen, dass strategisches Wählen nur infrage kommen kann, wenn die Chance (wie in dem obigen Beispiel) zugunsten eines Patts zwischen der dritt- und zweitliebsten Partei ausfällt. Bei einer 1:1-Chance oder weniger sollte immer die bevorzugte Partei gewählt werden. Aus welchem Grund? Weil der linke Bruch nicht kleiner als eins werden kann. Der Wähler bewertet per Definition seine bevorzugte Partei mindestens genauso hoch wie seine zweitliebste Partei. Die Differenz im Zähler wird daher immer größer oder gleich der Differenz im Nenner sein. Damit strategisch gewählt wird, muss also eine Situation vorliegen, in der ein Patt zwischen den beiden vom Wähler weniger präferierten Parteien wahrscheinlicher erscheint als ein Patt zwischen der bevorzugten Partei und der drittbesten Partei.

7 Dies ist ein anderer Zusammenhang als bei der Entscheidung zur Wahlteilnahme. Letztere hängt tatsächlich von der absoluten Wahrscheinlichkeit eines Patts ab (vgl. Riker u. Ordeshook 1968). 
Diese Situation liegt vor allem dann vor, wenn die bevorzugte Partei im Rennen um den Sieg an dritter Stelle liegt. Generell ist die Wahrscheinlichkeit eines unentschiedenen Wahlausgangs zwischen den beiden aussichtsreichsten Parteien immer größer als die Wahrscheinlichkeit, dass zwei andere Parteien gemeinsam auf dem ersten Platz landen (Myatt 2007). Je weiter eine drittplatzierte Partei also zurück liegt, umso geringer wird deren Chance, zusammen mit einer anderen Partei auf dem ersten Platz zu landen, und umso größer wird der Anreiz für Anhänger dieser Partei, ihre Stimme der zweitpräferierten Alternative (d.h. einer der beiden führenden Parteien) zu geben. Damit zeigt sich, dass das theoretische Modell in Ungleichung (2) eine präzise Abbildung der im zweiten Abschnitt beschriebenen Entscheidung zum strategischen Wählen bietet.

\subsection{Die statistische Schätzung strategischen Wählens}

Als nächstes wenden wir uns der statistischen Umsetzung des Modells zur Untersuchung strategischen Wählens anhand deutscher Wahlkreisergebnisse zu. Offenbar wird immer dann strategisch gewählt, wenn das Wahrscheinlichkeitsverhältnis in Ungleichung (2) größer ausfällt als das Nutzenverhältnis. Im Folgenden bezeichnen wir das Nutzenverhältnis der Einfachheit halber als ,Stärke der Präferenz' und das Wahrscheinlichkeitsverhältnis als ,strategischen Anreiz‘. Die Stärke der Präferenz eines Wählers für seine bevorzugte Partei ist dessen private Information. Sie bleibt außenstehenden Beobachtern in der Regel verborgen und wird auch in der vorliegenden Analyse als unbeobachtet angenommen. Der strategische Anreiz dagegen kann empirisch bestimmt werden. Er ist vom Wähler unabhängig und ergibt sich allein aus der Wahlkreissituation.

Das Wahlkriterium in Ungleichung (2) sagt uns, dass - gegeben die Stärke der Präferenz eines Wählers - dessen Entscheidung, strategisch zu wählen, nur von der Höhe des strategischen Anreizes in seinem Wahlkreis abhängt. Das heißt, auch wenn wir die Stärke der Präferenz der Wähler nicht kennen, so muss doch die Zahl der strategischen Wähler in einem Wahlkreis zunehmen, wenn der Anreiz, strategisch zu wählen, in diesem Wahlkreis steigt. Wenn also beispielsweise der Anreiz für PDS-Anhänger, strategisch die SPD zu wählen, im Wahlkreis zunimmt, so würden wir erwarten, dass die PDS im Wahlkreis Stimmen verliert und die SPD Stimmen hinzu gewinnt. Unter der Annahme, dass die Präferenzstärken der Wähler homogen über Wahlkreise hinweg verteilt sind, sich also nicht systematisch zwischen Wahlkreisen unterscheiden, lassen sich Unterschiede im strategischen Anreiz zwischen Wahlkreisen dazu nutzen, um strategisches Wählen zu bestimmen. Dazu müssen wir lediglich den strategischen Anreiz berechnen können, ein Maß für strategisches Wählen im Wahlkreis besitzen und ein statistisches Modell formulieren, mit dessen Hilfe sich auch das Ausmaß strategischen Wählens auf Wahlkreisebene nachvollziehen lässt. Jeder dieser Schritte wird im Folgenden erläutert.

Zur Berechnung des strategischen Anreizes benötigen wir ein Verfahren zur Bestimmung der Pattwahrscheinlichkeiten. Die übliche Vorgehensweise besteht darin, das Wahlergebnis als eine Zufallsvariable aufzufassen, die einer bestimmten Verteilung folgt (vgl. Hoffman 1982; Palfrey 1989). Diese Verteilung wird durch eine Dichtefunktion beschrieben. Um die Wahrscheinlichkeit eines Patts zu berechnen, muss die Dichte über alle möglichen Ergebnisse, bei denen zwei Parteien mit derselben Stimmenzahl auf dem ersten Platz enden, summiert (bzw. integriert) werden. Es könnten beispielsweise zwei 
Parteien mit je $40 \%$ der Erststimmen auf den ersten Platz gelangen oder auch mit je $45 \%$. Rein rechnerisch können zwei Parteien lediglich mit jeder Stimmenzahl zwischen einem Drittel und der Hälfte aller Stimmen im Wahlkreis auf dem ersten Platz landen. Die Wahrscheinlichkeit eines Patts beider Parteien ergibt sich schlicht aus der Summe der Einzelwahrscheinlichkeiten dieser möglichen Ergebnisse.

Im Folgenden wird auf ein elegantes und einfaches Verfahren zur Bestimmung der Pattwahrscheinlichkeiten zurückgegriffen, das von Myatt und Fisher (2002) entwickelt wurde. Myatt und Fisher schlagen vor, das voraussichtliche Wahlergebnis als eine dirichletverteilte Zufallsvariable aufzufassen. Die Dirichletverteilung ist naheliegend, da sie eigens zur Beschreibung der Wahrscheinlichkeit von Anteilswerten entwickelt wurde und es sich bei den Wahlergebnissen um ebensolche Anteilswerte (Stimmenanteile) handelt. ${ }^{8}$ Anhand dieser Verteilung zeigen die Autoren, dass die Integrale der einzelnen Pattwahrscheinlichkeiten einer bekannten Funktion folgen, der sogenannten unvollständigen Beta-Funktion. ${ }^{9}$ Die Berechnung dieser Funktion ist einfach und wird von den meisten Statistikprogrammen standardmäßig durchgeführt. Auf eine Herleitung des Ergebnisses von Myatt und Fisher wird hier aus Platzgründen verzichtet. ${ }^{10}$

Im Folgenden notieren wir mit $I_{x y}$ den , strategischen Anreiz eines Anhängers von Partei $x$, seine Stimme der von ihm zweitpräferierten Partei $y$ zu geben'. Unter der Annahme einer Dirichletverteilung des Wahlergebnisses lässt sich der strategische Anreiz aus Ungleichung (2) dann folgendermaßen ausdrücken:"

$$
\begin{aligned}
I_{x y} & \equiv \log \frac{2 p_{x y}+p_{y z}}{2 p_{x y}+p_{x z}} \\
& =\log \frac{B_{1 / 3}\left(1+\pi_{z} s, 1+\left(1-\pi_{z}\right) s\right)+2^{\left(\pi_{x}-\pi_{z}\right) s-1} B_{1 / 3}\left(1+\pi_{x} s, 1+\left(1-\pi_{x}\right) s\right)}{B_{1 / 3}\left(1+\pi_{z} s, 1+\left(1-\pi_{z}\right) s\right)+2^{\left(\pi_{Y}-\pi_{z}\right) s-1} B_{1 / 3}\left(1+\pi_{y} s, 1+\left(1-\pi_{y}\right) s\right)} .
\end{aligned}
$$

Dabei bezeichnet $B_{1 / 3}(\cdot)$ die unvollständige Beta-Funktion bis zur Stelle 1/3 und $\pi_{x}, \pi_{y}$ und $\pi_{z}$ die Anteilswerte der Anhängerschaft der vom Wähler bevorzugten Partei sowie seiner zweit- und drittpräferierten Partei. Die drei Anteilswerte - die sich notwendigerweise zu eins addieren - geben an, wie viele Anhänger die jeweiligen Parteien in der Wählerschaft haben. Auf der Basis dieser Werte sowie eines zusätzlichen Parameters $s$ werden die Pattwahrscheinlichkeiten und damit der Anreiz zum strategischen Wählen berechnet.

Der Parameter $s$ in Gleichung (3) ist ein Maß für die Unsicherheit des Wählers bezüglich der Anhängerschaft der einzelnen Parteien im Wahlkreis. Je kleiner $s$, desto größer ist

8 Generell wird die Dirichletverteilung verwendet, um Zufallsvariablen mit mehr als zwei Ausprägungen zu modellieren, wobei letztere stets positive Werte annehmen müssen, die in der Summe 1 ergeben.

9 Die unvollständige Beta-Funktion bis zur Stelle $g \in[0,1]$ mit Parametern $a$ und $b$ ist das Integral: $B_{g}(a, b)=\int_{0}^{g} t^{a-1}(1-t)^{b-1} d t$.

10 Der interessierte Leser sei verwiesen auf Myatt und Fisher (2002, S. 30-31) oder Herrmann $(2008$, S. 31).

11 Aus rechnerischen Gründen wird im Folgenden mit dem Logarithmus des Verhältnisses der Pivot-Wahrscheinlichkeiten gearbeitet. Inhaltlich ändert sich dadurch nichts, weshalb wir weiter vom ,strategischen Anreiz's sprechen werden. 
die Unsicherheit des Wählers und desto kleiner der Anreiz zum strategischen Wählen. In einer früheren Studie hat Herrmann (2008) die Unsicherheit ostdeutscher Wähler bei den letzten Bundestagswahlen geschätzt und nachgewiesen, dass Wähler sehr unsicher bezüglich der Verteilung der Parteianhängerschaften in ihrem Wahlkreis sind. Dieses Ergebnis leuchtet ein angesichts der Tatsache, dass Meinungsumfragen auf Wahlkreisebene unüblich sind. Zur Berechnung des strategischen Anreizes wird auf die Schätzwerte aus dieser Studie zurückgegriffen. ${ }^{12}$

Als Maß für die Anhängerschaften der drei Parteien werden die im Wahlkreis vergebenen Zweitstimmen für PDS, CDU und SPD verwendet. Für einen Anhänger der PDS beispielsweise ergäbe sich die Anhängerschaft seiner bevorzugten Partei als

$$
\pi=\frac{\text { Stimmen PDS }}{\text { Stimmen PDS }+ \text { Stimmen CDU + Stimmen SPD }} .
$$

Analog dazu lassen sich die Anteilswerte der anderen beiden Parteien berechnen. Zusammen bestimmen diese Anteilswerte die Höhe des strategischen Anreizes für Anhänger aller drei Parteien. Je nachdem, welche Wähler betrachtet werden, ordnet man jenen bestimmte Anteilswerte zu. Um zum Beispiel den strategischen Anreiz eines SPD-Anhängers mit Zweitpräferenz CDU zu berechnen, weist man $\pi_{x}$ den Anteilswert der SPD, $\pi_{y}$ den der CDU und $\pi_{z}$ den der PDS zu. Um den Anreiz eines SPD-Anhängers mit Zweitpräferenz PDS zu berechnen, weist man $\pi_{x}$ den Anteilswert der SPD, $\pi_{y}$ den der PDS und $\pi_{z}$ den der CDU zu, usw. Auf diese Weise lässt sich der strategische Anreiz im Wahlkreis für jeden Wählertyp berechnen. ${ }^{13}$

Was rechtfertigt die Verwendung des Zweitstimmenergebnisses zur Berechnung der Anhängerschaften im Wahlkreis? Zweitstimmen werden proportional auf Bundesebene in Sitze umgerechnet. Dadurch entstehen keine Anreize zum strategischen Wählen wie bei der Erststimme. Natürlich ist nicht auszuschließen, dass auch mit der Zweitstimme strategisch gewählt wird, etwa in Form von Leihstimmen (vgl. Schoen 1998; Pappi u. Thurner 2002). Derartiges, unehrliches‘ Zweitstimmenwählen würde jedoch auf die Beeinflussung des nationalen Wahlergebnisses abzielen und demnach in allen Wahlkreisen gleichermaßen auftreten. Entsprechend wären Unterschiede im Zweitstimmenwählen zwischen Wahlkreisen immer noch das Resultat unterschiedlicher Wählerpräferenzen in den einzelnen Wahlkreisen. Aus diesem Grund soll an der Verteilung der Zweitstimmen im Wahlkreis als Maß für die tatsächlichen Anhängerschaften der Parteien im Wahlkreis vorerst festgehalten werden. Zur weiteren Prüfung des Einflusses nicht-ehrlichen Zweitstimmenwählens werden alle Ergebnisse überdies einer Sensitivitätsanalyse unterzogen, die im Verlauf des nächsten Abschnitts erläutert wird.

12 Die von Herrmann (2008) geschätzten Werte sind: 1994, $s=3,8 ; 1998, s=7,55 ; 2002, s=5,04$ und 2005, $s=1,43$. Auch für die Bundestagswahl 2009 wird angenommen, dass die Wähler sehr unsicher bezüglich der Anhängerschaften in ihrem Wahlkreis waren, und $s=1$ gesetzt. Andere Werte für $s$ führen zu keiner wesentlichen Änderung der Ergebnisse.

13 Es sei angemerkt, dass in der folgenden Analyse nicht angenommen wird, Wähler würden die genaue Verteilung der Parteianhängerschaften in ihrem Wahlkreis kennen. Wie oben bereits erwähnt, wird im Gegenteil davon ausgegangen, dass Wähler nur geringe Kenntnis dieser Verteilung besitzen und daher eher unsicher sind und wenig geneigt, strategisch zu wählen. 
Um die Bedeutung strategischer Anreize auf das Wahlverhalten bestimmen zu können, benötigt man ein $\mathrm{Ma}$ für strategisches Wählen. Hier wird im Einklang mit früheren Studien die Differenz zwischen den Erst- und Zweitstimmen einer Partei im Wahlkreis verwendet (vgl. Cox 1997; Bawn 1999; Gschwend et al. 2003). Der Grund dafür ist naheliegend: Profitiert eine Partei von strategischen Stimmen, so sollte sich dies positiv auf ihre Erst-Zweitstimmendifferenz auswirken; verliert sie Stimmen aufgrund von strategischem Wählen, sollte sich dies negativ auf ihre Stimmendifferenz auswirken. Sofern also im Wahlkreis strategisch gewählt wird, sollte sich dies in den Differenzen der abgegebenen Erst- und Zweitstimmen der Parteien niederschlagen.

Zur Modellierung strategischer Wählerbewegungen genügt es nicht, die Stimmendifferenzen der Parteien isoliert voneinander zu betrachten, denn eine strategische Stimme hat immer zwei Auswirkungen: Sie reduziert die Stimmenzahl einer Partei und vergrößert gleichzeitig die Stimmenzahl einer anderen Partei. Steigt also die Zahl der PDS-Anhänger, die sich strategisch für die SPD entscheiden, so sollte die Stimmendifferenz der PDS sinken, die Stimmendifferenz der SPD jedoch um denselben Betrag steigen. Ähnliches gilt für strategische Wanderbewegungen zwischen den anderen Parteien.

Formal lassen sich sämtliche Wanderbewegungen im Wahlkreis in dem folgenden Gleichungssystem zusammenfassen:

$$
\begin{aligned}
& S 1-S 2=\psi_{C S} I_{C S}+\psi_{P S} I_{P S}+0 \\
& C 1-C 2=-\psi_{C S} I_{C S}+0+\psi_{P C} I_{P C} \\
& P 1-P 2=00-\psi_{P S} I_{P S}-\psi_{P C} I_{P C}
\end{aligned}
$$

Dabei bezeichnet $I_{C S}$ den strategischen Anreiz eines CDU-Anhängers, seine Stimme der SPD zu geben, $I_{P S}$ den Anreiz eines PDS-Anhängers, die SPD zu wählen, und $I_{P C}$ den Anreiz eines PDS-Anhängers, die CDU zu wählen. Wie man sieht, ist jede der drei strategischen Anreizvariablen mit einem eigenen Koeffizienten $\left(\psi_{C S}, \psi_{P S}, \psi_{P C}\right)$ versehen, der die Zahl der Wähler angibt, die aufgrund des strategischen Anreizes ihre zweitpräferierte Partei wählen. Da dies impliziert, dass Stimmen von einer Partei zur anderen wandern, taucht jeder Koeffizient einmal mit einem positiven und einmal mit einem negativen Vorzeichen im Gleichungssystem auf. Sofern also 1000 PDS-Anhänger strategisch der CDU ihre Stimme geben, so hat dies einen positiven Effekt auf die Stimmendifferenz der CDU und einen negativen Effekt auf die Stimmendifferenz der PDS. Dem Betrag nach müssen beide Effekte jedoch gleich (nämlich 1000) sein, und genau das wird mit der Spezifikation in Gleichungen (4) erreicht.

Auf den ersten Blick könnte es so erscheinen, als würden in Gleichungen (4) drei Typen von Wählern ausgeschlossen, nämlich SPD-Anhänger mit Zweitpräferenz CDU sowie SPD- und CDU-Anhänger mit Zweitpräferenz PDS. Diese Wählertypen sind allerdings implizit im Modell enthalten, denn mathematisch ist der strategische Anreiz beispielsweise eines CDU-Anhängers, die SPD zu wählen, einfach das Gegenteil des Anreizes eines SPD-Anhängers, die CDU zu wählen; dem entspricht formal: $I_{C S}=-I_{S C}$. Dasselbe gilt für die strategischen Anreize zwischen den anderen Parteien, d. h. $I_{P S}=-I_{S P}$ und $I_{P C}=-I_{C P} \cdot{ }^{14}$ Mit anderen Worten: Steigt der Anreiz für PDS-Anhänger, im Wahlkreis

14 Es ist einfach, diesen Zusammenhang zu zeigen (vgl. Herrmann 2008). 
die CDU zu wählen, um eine Einheit, dann sinkt gleichzeitig der Anreiz für CDU-Anhänger, die PDS zu wählen, um eine Einheit. Zur Modellierung der Wählerströme zwischen den Parteien im Wahlkreis reichen daher drei Anreizvariablen aus.

Zur Schätzung der Parameter in Gleichungen (4) wird angenommen, dass die Stimmendifferenzen der Parteien von Zufallsfehlern behaftet sind. ${ }^{15} \mathrm{Außerdem}$ werden in jede Gleichung Konstanten eingefügt, da nicht angenommen werden kann, dass die Stimmendifferenzen der Parteien ohne strategisches Wählen null betragen. Die Konstanten dienen lediglich der Kalibrierung des Modells. Ihnen kommt keine inhaltliche Bedeutung zu. Insgesamt ergibt sich das vollständige statistische Modell als

$$
\begin{aligned}
& S 1-S 2=\alpha_{S}+\psi_{C S} I_{C S}+\psi_{P S} I_{C S}+0+\varepsilon_{S} \\
& C 1-C 2=\alpha_{C}-\psi_{C S} I_{C S}+0+\psi_{P C} I_{P C}+\varepsilon_{C} \\
& P 1-P 2=\alpha_{P}+0-\psi_{P S} I_{P S}-\psi_{P C} I_{P C}+\varepsilon_{P} .
\end{aligned}
$$

Dieses Gleichungssystem kann mit der OLS-Methode geschätzt werden (vgl. Greene 2003, S.343). Um die positiven, negativen sowie die Null-Zusammenhänge zwischen den strategischen Anreizvariablen und den Stimmendifferenzen zu gewährleisten, wurden die Anreizvariablen jeweils mit Indikator-Variablen mit den Werten $-1,0$ oder 1 multipliziert.

\section{Ergebnisse}

Tabelle I zeigt den geschätzten Einfluss der Wahlkreissituation auf strategisches Wählen. Bezüglich der Interpretation sei vorweg geschickt, dass die geschätzten Effekte der strategischen Anreizvariablen nur innerhalb einer Wahl und nicht über Wahlen hinweg vergleichbar sind. ${ }^{16}$ Ein positiver Effektkoeffizient bedeutet, dass mit steigendem strategischem Anreiz mehr Wähler von ihrer bevorzugten Partei Abstand nehmen und ihre Stimme stattdessen der zweitpräferierten Partei geben. Ein negativer Koeffizient bedeutet dagegen, dass mit steigendem Anreiz weniger Wähler ihre zweitpräferierte Partei wählen, was offensichtlich der theoretischen Erwartung widerspricht.

Wie man anhand von Tab. 1 sieht, sind ostdeutsche Wähler empfänglich für strategische Anreize in ihrem Wahlkreis. Dies trifft vor allem auf Anhänger der PDS (ab 2007: DIE LINKE) zu. Die Koeffizienten der strategischen Anreizvariablen für diese Wähler sind durchweg positiv und - mit Ausnahme der Koeffizienten für 1994 und 2009 - auch statistisch signifikant verschieden von null. Im Klartext bedeutet das, wenn sich beispielsweise im Jahr 1994 der strategische Anreiz eines PDS-Anhängers, die CDU zu wählen, um eine Einheit erhöht hat, dann haben im Mittel 11.435 PDS-Anhänger im jeweiligen Wahlkreis ihre Stimme strategisch der CDU gegeben. Im Gegensatz dazu fallen die Effekte für den strategischen Anreiz, zwischen CDU und SPD zu wählen, deutlich geringer aus.

15 Genauer gesagt, wird davon ausgegangen, dass die Fehlerterme der Stimmendifferenzen einer multivariaten Normalverteilung mit Mittelwert null und Varianz $\sigma$ folgen.

16 Da für jede Wahl andere Werte des Unsicherheitsparameters $s$ angenommen wurden, sind die strategischen Anreizvariablen für jede Wahl unterschiedlich skaliert. 
Tab. 1: Strategisches Wählen in ostdeutschen Wahlkreisen. (Quelle: eigene Berechnungen)

\begin{tabular}{llllll}
\hline & 1994 & 1998 & 2002 & 2005 & 2009 \\
\hline Strategischer Anreiz CDU $\leftrightarrow$ SPD & 224 & -3747 & 2825 & -5641 & 10604 \\
& $(2461)$ & $(2919)$ & $(3878)$ & $(9972)$ & $(12703)$ \\
Strategischer Anreiz PDS $\leftrightarrow$ SPD & 3160 & $16054^{*}$ & $16305^{*}$ & $58034^{*}$ & 24127 \\
& $(2416)$ & $(5135)$ & $(4736)$ & $(15093)$ & $(14196)$ \\
Strategischer Anreiz PDS $\leftrightarrow$ CDU & $11453^{*}$ & $10310^{*}$ & $7337^{*}$ & $50441^{*}$ & $43092^{*}$ \\
& $(2073)$ & $(2115)$ & $(1818)$ & $(10897)$ & $(11089)$ \\
Konstante SPD & 268 & 150 & $-6562^{*}$ & $854^{*}$ & $4948^{*}$ \\
& $(630)$ & $(1149)$ & $(1584)$ & $(395)$ & $(813)$ \\
Konstante CDU & $-806^{*}$ & $1838^{*}$ & 1371 & $5327^{*}$ & $2305^{*}$ \\
& $(385)$ & $(563)$ & $(726)$ & $(415)$ & $(732)$ \\
Konstante PDS & $3485^{*}$ & $5640^{*}$ & $10105^{*}$ & $1479^{*}$ & -267 \\
& $(916)$ & $(1367)$ & $(1479)$ & $(562)$ & $(694)$ \\
$\mathrm{R}^{2}$ & 0,46 & 0,67 & 0,59 & 0,64 & 0,55 \\
$\mathrm{~N}$ & 213 & 210 & 171 & 168 & 141 \\
\hline
\end{tabular}

Tabelleneinträge sind OLS-Koeffizienten. Standardfehler, korrigiert für Abhängigkeit innerhalb von Wahlkreisen, in Klammern. Ab 2007 steht PDS stellvertretend für DIE LINKE.

${ }^{*} p<0,05$ (zweiseitiger Test)

In jedem Wahljahr ist der entsprechende Koeffizient um ein vielfaches geringer als die beiden anderen Koeffizienten und in keinem Fall ist der Koeffizient signifikant verschieden von null. Das heißt, es lässt sich nicht mit hinreichender Verlässlichkeit (konkret: mit einer Irrtumswahrscheinlichkeit von weniger als $5 \%$ ) sagen, ob zwischen beiden Parteien überhaupt strategisch gewählt wird. Wenn dies im Wahlkreis aber der Fall ist, dann ist immer die PDS/DIE LINKE davon betroffen.

Insgesamt gibt es für jede der vier Bundestagswahlen klare Anzeichen von strategischem Wählen. Allerdings zeigen nur Anreize zum Stimmenwechsel zwischen PDS/DIE LINKE und einer der beiden anderen Parteien eine Wirkung auf die Wähler. Dies könnte zweierlei Ursachen haben: Einerseits könnte die Zahl der Wähler, die die PDS/DIE LINKE als schlechteste Partei beurteilt (d.h. Wähler, die die CDU bevorzugen und die SPD als zweitbeste Partei sehen oder umgekehrt) vergleichsweise gering sein, sodass sich strategisches Wählen statistisch nicht bemerkbar macht. Andererseits könnte es sein, dass dieser Wählertyp seine präferierte Partei besonders stark gegenüber der zweitpräferierten Alternative bevorzugt. Beide Möglichkeiten sind denkbar und schließen sich nicht aus. Wie es dazu kommt, dass ein Wähler mit CDU und SPD auf Erst- und Zweitpräferenz eine andere Bewertung der Alternativen vornimmt als die übrigen Wähler, darüber kann an dieser Stelle nur spekuliert werden.

Trotz der ansonsten klaren Ergebnisse muss bei der Interpretation zunächst noch eine Einschränkung vorgenommen werden. Die bisherige Analyse beruht gänzlich auf der Annahme, die lokale Verteilung der Parteipräferenzen lasse sich aus dem Zweitstimmenergebnis im Wahlkreis ablesen. Letztlich können wir jedoch nicht davon ausgehen, dass Wähler mit der Zweitstimme immer ehrlich wählen. Beispielsweise könnten sich Anhänger von CDU bzw. SPD im Hinblick auf die spätere Regierungsbildung entschließen, ihre Stimme dem designierten Koalitionspartner FDP bzw. den Grünen zu geben (vgl. Pappi u. 
Thurner 2002; Gschwend 2007; Shikano et al. 2009). In der Summe hätte dies zur Folge, dass wir die Anhängerschaft der CDU und SPD systematisch geringer einschätzen als sie tatsächlich ist. Zwar sollte dieser Effekt alle Wahlkreise gleichermaßen betreffen, sodass Unterschiede in der Zweitstimmenverteilung zwischen Wahlkreisen nach wie vor Unterschiede in den Wählerpräferenzen widerspiegeln. Eine mögliche Verzerrung der Ergebnisse lässt sich jedoch nicht völlig ausschließen.

Um einen Eindruck davon zu gewinnen, wie groß die Verzerrung ausfallen kann, nehmen wir an, es gäbe kein strategisches Erststimmenwählen und alle Wähler würden mit der Erststimme ehrlich wählen. Sofern sich Anhänger von CDU oder SPD nun entschlieBen, ihre Zweitstimme der FDP bzw. den Grünen zu geben, sollte dies zu einem Überschuss an Zweitstimmen (gegenüber den Erststimmen) für diese Parteien führen. Eine mögliche Korrektur der Analyse bestünde dann darin, die überzähligen Zweitstimmen für die FDP rückwirkend der CDU anzurechnen und überzählige Zweitstimmen der Grünen der SPD zuzuschlagen. Falls also die bisherigen Ergebnisse allein auf nicht-ehrliches Zweitstimmenwählen zurückgehen, sollten sich nach dieser Korrektur keine Anzeichen mehr für strategisches Erststimmenwählen finden lassen.

Tabelle 2 zeigt, dass dem nicht so ist: Im Einklang mit den Ergebnissen in Tab. 1 ergibt sich erneut ein signifikanter Einfluss der strategischen Anreizvariablen auf die Wahl zwischen PDS/DIELINKE und SPD bzw. PDS/DIELINKE und CDU. Anreize für eine strategische Entscheidung zwischen SPD und CDU haben erneut keinen statistisch signifikanten Effekt. Im Vergleich zu Tab. 1 erreicht der Koeffizient für eine strategische Entscheidung zwischen PDS und SPD im Jahr 1994 nun statistische Signifikanz. Einen Beleg für die These, dass strategisches Zweitstimmenwählen zwar vorkommt, davon aber ein

Tab. 2: Robustheits-Check: Strategisches Wählen in ostdeutschen Wahlkreisen unter Berücksichtigung von koalitionsbezogenem Zweitstimmenwählen. (Quelle: eigene Berechnungen)

\begin{tabular}{llllll}
\hline & 1994 & 1998 & 2002 & 2005 & 2009 \\
\hline Strategischer Anreiz CDU $\leftrightarrow$ SPD & -1919 & -5354 & 1957 & -8753 & 7198 \\
& $(2527)$ & $(3201)$ & $(4432)$ & $(11447)$ & $(13669)$ \\
Strategischer Anreiz PDS $\leftrightarrow$ SPD & $5093^{*}$ & $17527^{*}$ & $20053^{*}$ & $38875^{*}$ & 13795 \\
& $(2557)$ & $(5652)$ & $(4688)$ & $(16315)$ & $(15242)$ \\
Strategischer Anreiz PDS $\leftrightarrow$ CDU & $12198^{*}$ & $10996^{*}$ & $6427^{*}$ & $46576^{*}$ & $39857^{*}$ \\
& $(2261)$ & $(2240)$ & $(2085)$ & $(11839)$ & $(13910)$ \\
Konstante SPD & -64 & -1076 & $-9341^{*}$ & -902 & $3220^{*}$ \\
& $(659)$ & $(1384)$ & $(1707)$ & $(503)$ & $(1031)$ \\
Konstante CDU & $-1416^{*}$ & 666 & 945 & 96 & -1382 \\
& $(417)$ & $(644)$ & $(839)$ & $(385)$ & $(724)$ \\
Konstante PDS & $3976^{*}$ & $6400^{*}$ & $11285^{*}$ & $1989^{*}$ & 679 \\
& $(989)$ & $(1535)$ & $(1515)$ & $(736)$ & $(769)$ \\
$\mathrm{R}^{2}$ & 0,45 & 0,61 & 0,63 & 0,28 & 0,34 \\
$\mathrm{~N}$ & 213 & 210 & 171 & 168 & 141 \\
\hline
\end{tabular}

Tabelleneinträge sind OLS-Koeffizienten. Standardfehler, korrigiert für Abhängigkeit innerhalb von Wahlkreisen, in Klammern. Ab 2007 steht PDS stellvertretend für DIE LINKE.

${ }^{*} p<0,05$ (zweiseitiger Test) 
Abb. 1: Vorhergesagte strategische Wählerwanderungen. Punkte geben die Zahl der strategischen Wähler an, die in einem Wahlkreis von einer Partei zu einer anderen gewechselt sind. Aus Gründen der Übersichtlichkeit sind in den einzelnen Teilgrafiken nicht alle Punkte auf einer Linie, sondern untereinander abgetragen.

(Quelle: eigene Berechnungen)
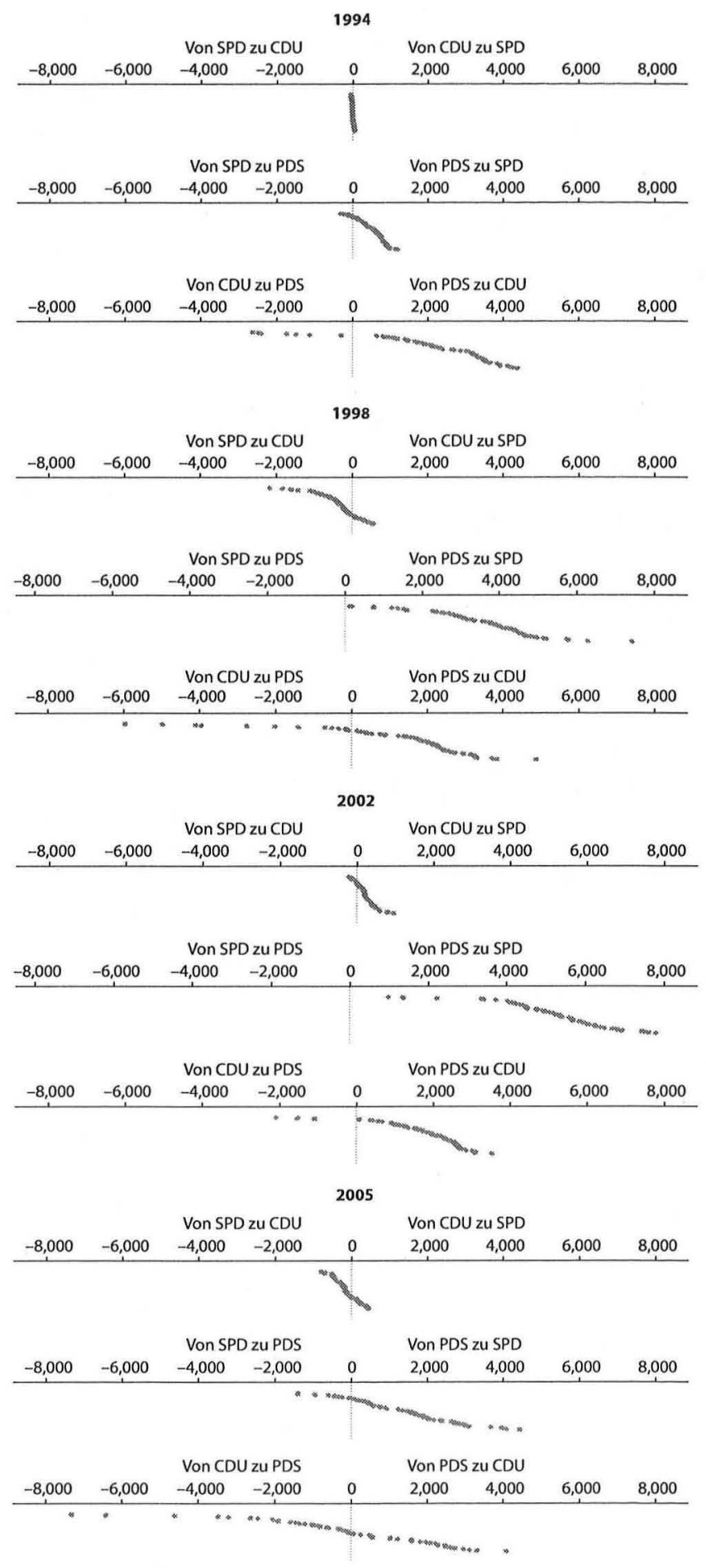
Abb. 1: (Fortsetzung)

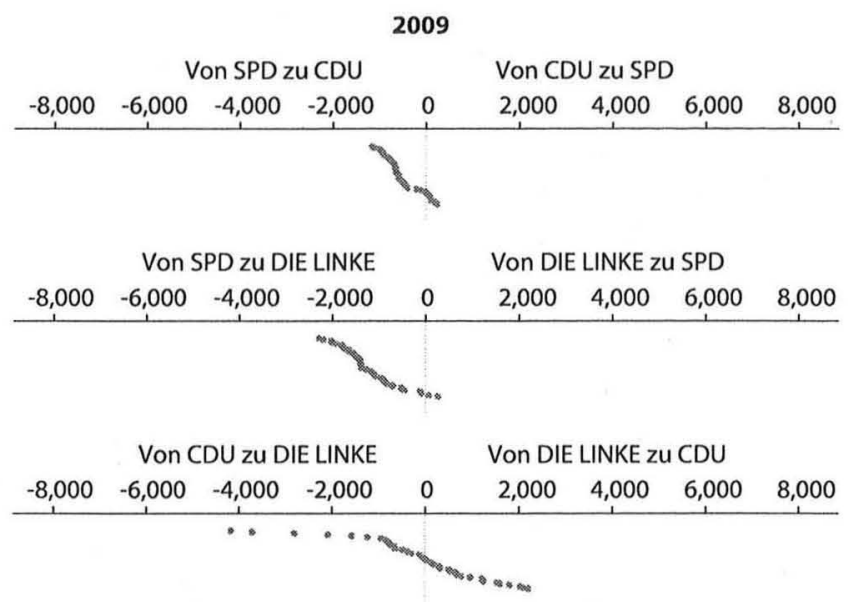

uniformer Effekt ausgeht, der mit strategischen Anreizen bei der Erststimme nicht korreliert, könnte man darin sehen, dass die Konstanten für SPD und CDU in Tab.2 meist insignifikant sind. Insgesamt gibt es also nach wie vor deutliche Anzeichen für strategisches Wählen in allen betrachteten Bundestagswahlen. Nachdem wir nun wissen, dass in Ostdeutschland strategisch gewählt wird, stellt sich die Frage, welche Parteien am meisten von strategischem Wählen profitierten. Tabelle 1 gibt über die Richtung strategischen Wählens keinen Aufschluss, sondern lediglich darüber, ob es bedeutsame Wählerwanderungen zwischen Parteien gibt. ${ }^{17}$ Auf der Basis der Ergebnisse in Tab. I werden deshalb für jeden Wahlkreis die vorhergesagten strategischen Wählerwanderungen zwischen zwei Parteien berechnet. Abbildung 1 gibt diese Vorhersagen wieder.

Offensichtlich gibt es für jede Partei sowohl Gewinne als auch Verluste - eine Tatsache, die sich aus Tab. I nicht ablesen lässt. Doch sind diese nicht gleich über alle Parteien verteilt. Insgesamt profitiert die SPD am meisten von strategischem Wählen. Vor allem in den Wahlen von 1998 bis 2005 gingen viele Stimmen von PDS-Anhängern in ostdeutschen Wahlkreisen an die SPD. Interessanterweise gingen 1994, im Jahr der letzten Wiederwahl Kohls, die meisten strategischen Stimmen von PDS-Anhängern an die CDU. Dies ist ein erster Hinweis darauf, dass PDS-Anhänger einen entscheidenden Einfluss auf die Sitzverteilung im Bundestag gehabt haben können. Im Jahr 2009 gingen erneut die meisten strategischen Stimmen von DIE LINKE-Anhängern an die CDU, allerdings so gut wie keine mehr an die SPD, sondern im Gegenteil: Nahezu alle strategischen Stimmentransfers zwischen SPD und DIE LINKE gingen 2009 zulasten der SPD. Ungeachtet ihrer generellen Verluste profitierte somit auch die PDS/DIE LINKE verschiedentlich von strategischen Stimmen, insbesondere in den beiden jüngsten Bundestagswahlen.

Bevor wir uns der Frage der Sitzverteilung im Bundestag zuwenden, wollen wir noch einen Blick auf das gesamte Ausmaß strategischen Wählens in den einzelnen Wahlkreisen werfen. Dazu wird auf der Basis der vorhergesagten Stimmentransfers für jeden Wahlkreis die absolute Zahl von Wählern, die ihre Stimme einer anderen Partei als der bevorzugten geben, berechnet und diese dann ins Verhältnis zur Zahl der insgesamt im Wahlkreis

17 Dies liegt an der im vorherigen Kapitel beschriebenen Symmetrie des strategischen Anreizes. 
Abb. 2: Anteil strategischer Wähler im Wahlkreis. Punkte geben den Anteil strategischer Stimmen an allen gültigen Erststimmen im Wahlkreis an. Aufgrund von Wahlkreisneueinteilungen können für manche Wahlkreise nur Werte zu einem oder zwei Jahren präsentiert werden. Speziell im Jahr 2009 mussten einige Ost-Berliner Wahlkreise ausgeschlossen werden, für die aufgrund der Stärke der Grünen die Modellbedingung eines Dreiparteienwettbewerbs nicht gerechtfertigt erschien. (Quelle eigene Berechnungen)

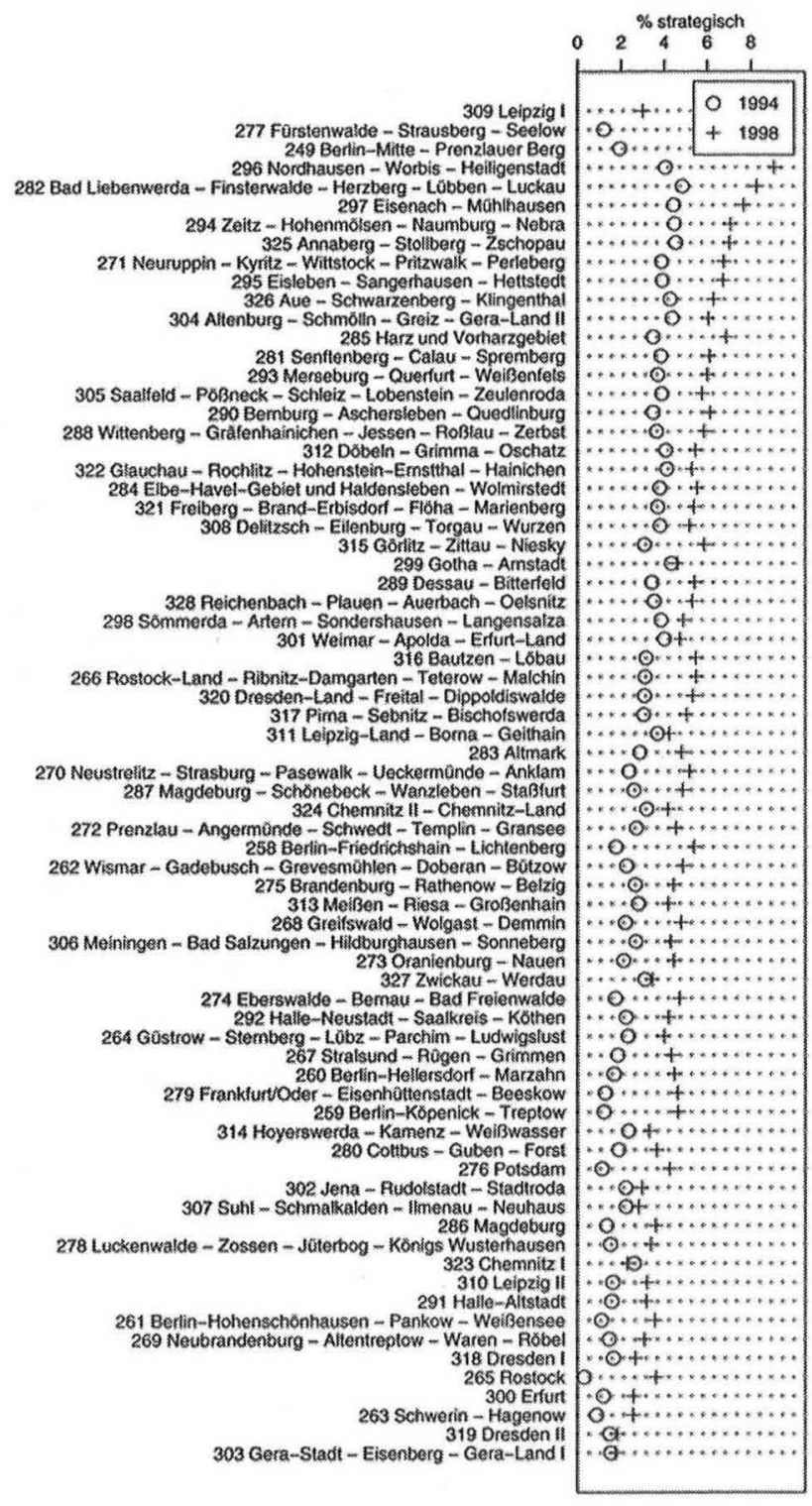

abgegebenen Stimmen gesetzt. Die so vorhergesagten Anteile strategischer Wähler im Wahlkreis sind in Abb. 2 dargestellt.

Zunächst sieht man, dass eine deutliche Varianz im strategischen Wählen über Wahlkreise hinweg besteht. Das liegt in erste Linie an den unterschiedlichen Ausgangsbedingungen: Da der Anreiz, strategisch zu wählen, nicht in jedem Wahlkreis gleich hoch ist, sollte auch der Anteil strategischer Wähler variieren. Weiterhin geht aus Abb. 2 hervor, dass 1998 und 2002 mehr strategische Erststimmen abgegeben wurden als in den anderen Wahljahren. Während 1998 und 2002 zwischen 2 und 9\% aller Stimmen im Wahlkreis 
Abb. 2: (Fortsetzung)

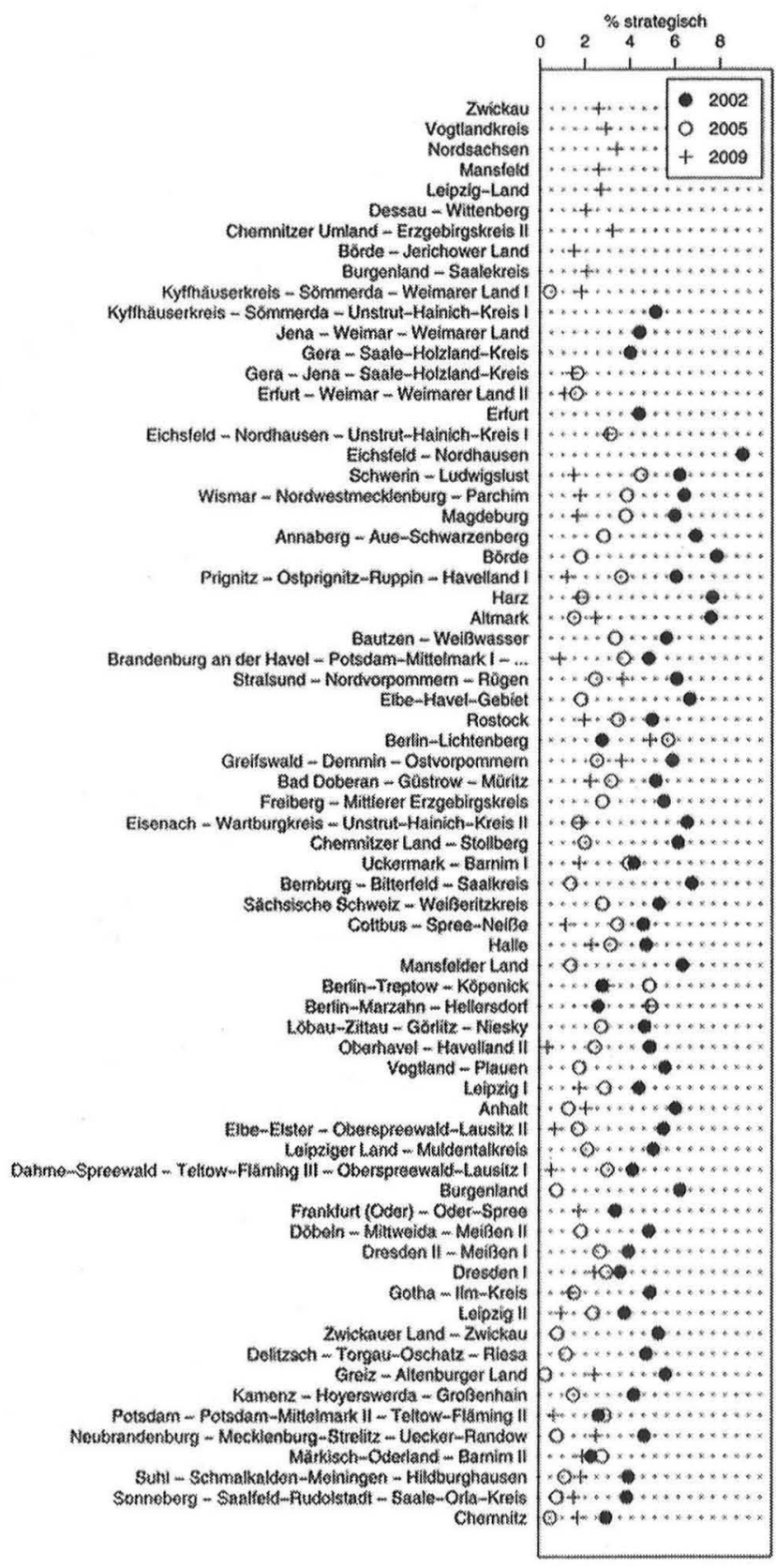


strategisch waren (im Durchschnitt jeweils etwa 4,5\%), wurden 1994, 2005 und 2009 nur bis zu 6\% strategische Stimmen abgegeben (im Durchschnitt jeweils etwa 2,8, 2,3 und $2,1 \%)$. Diese Zahlen machen deutlich, dass strategisches Wählen in Ostdeutschland bisher kein Massenphänomen darstellt. Dennoch können in einem knappen Rennen bereits ein paar Stimmen ausreichen, um die Wahl zu entscheiden.

Zuletzt wenden wir uns deshalb der Frage zu, welchen Einfluss strategisches Wählen auf die Sitzverteilung im Bundestag hatte. Dazu werden in einem ersten Schritt die Wahlkreise identifiziert, in denen die vorhergesagte Zahl strategischer Stimmen für mindestens eine der drei Parteien größer ist als der Stimmenvorsprung des Wahlkreissiegers vor dem zweitplatzierten Kandidaten. Alle Wahlkreise, die dieses Kriterium erfüllen, sind in Tab. 3 aufgelistet.

Wie man sieht, gibt es eine ganze Reihe von Wahlkreisen, die nur mit einem knappen Vorsprung entschieden wurden. In den meisten dieser Wahlkreise belegten CDU- und SPD-Kandidaten die ersten beiden Plätze. Der Stimmenvorsprung des Siegers vor dem Zweitplatzierten ist in der sechsten Spalte angegeben. In den darauf folgenden Spalten sind die jeweils vorhergesagten strategischen Stimmentransfers zwischen den Parteien aufgeführt. In der letzten Spalte ist der Vorsprung des Wahlkreisgewinners wiedergegeben, den jener/jene ohne die strategischen Stimmen von Anhängern anderer Parteien erzielt hätte. ${ }^{18}$ Sofern der Stimmenvorsprung des Wahlsiegers ohne strategisches Wählen negativ ausfällt (d.h. der Sieger ohne strategisches Wählen weniger Stimmen erhält als der Zweitplatzierte), ist dies ein Anzeichen dafür, dass strategisches Wählen den Wahlausgang im Wahlkreis entschieden hat.

Von 1994 bis 2009 war dies immerhin fünf Mal der Fall: Bei der Bundestagswahl 1994 gewann die CDU durch strategische Stimmen von PDS-Anhängern einen zusätzlichen Wahlkreis in Mecklenburg-Vorpommern. Ohne diesen zusätzlichen Wahlkreis hätte die CDU in Mecklenburg-Vorpommern nur eines von zwei Überhangmandaten errungen. ${ }^{19}$ Vier Jahre später beim Wahlsieg Gerhard Schröders profitierte die SPD von den Stimmen der PDS-Anhänger im Wahlkreis. Insgesamt wurden drei Wahlkreise mithilfe strategischer PDS-Anhänger gewonnen, was der SPD zwei Überhangmandate in MecklenburgVorpommern bescherte und der CDU ein Überhangmandat in Sachsen verwehrte. Im Jahr 2002 schließlich gewann die SPD dank strategischer Stimmen von PDS-Anhängern einen zusätzlichen Wahlkreis und damit ein Überhangmandat in Sachsen-Anhalt. In späteren Bundestagswahlen hatte strategisches Wählen keinen Einfluss mehr auf den Ausgang der Wahl im Wahlkreis.

18 Zur Berechnung dieses ,Nettovorsprungs‘ werden vom Stimmenvorsprung des Siegers alle strategischen Stimmen, die zu dessen Gunsten gingen, abgezogen und alle strategische Stimmen, die zugunsten des Zweitplatzierten gingen, hinzu addiert.

19 Diese und die folgenden Schlussfolgerungen beruhen auf den Angaben zur Sitzzuteilung der Parteien nach Ländern, wie sie offiziellen Quellen, etwa den Veröffentlichungen des Bundeswahlleiters (http://www.bundeswahlleiter.de/de/bundestagswahlen/fruehere_bundestagswahlen/; Zugegriffen am 16. Februar 2010), zu entnehmen sind. 
Tab. 3: Hat strategisches Wählen den Ausschlag gegeben? Vorhergesagte Stimmentransfers in knappen Wahlkreisenª (Quelle: eigene Berechnungen)

\begin{tabular}{|c|c|c|c|c|c|c|c|c|c|}
\hline \multirow[b]{2}{*}{ Jahr } & \multirow[b]{2}{*}{ Bundesland } & \multirow[b]{2}{*}{ Wahlkreis } & \multirow[b]{2}{*}{ Sieger } & \multirow[b]{2}{*}{ Zweiter } & \multirow{2}{*}{$\begin{array}{l}\text { Stimmen- } \\
\text { vorsprung }\end{array}$} & \multicolumn{3}{|c|}{ Strat. Stimmentransfers ${ }^{\mathrm{b}}$} & \multirow{2}{*}{$\begin{array}{l}\text { Netto- } \\
\text { vorsprung }\end{array}$} \\
\hline & & & & & & $\mathrm{C} \rightarrow \mathrm{S}$ & $\mathrm{P} \rightarrow \mathrm{S}$ & $\mathrm{P} \rightarrow \mathrm{C}$ & \\
\hline \multirow[t]{5}{*}{$\overline{1994}$} & Mecklenburg-V. & 262 Wismar/Gadebusch/... & $\overline{C D U}$ & SPD & 793 & -3 & 537 & 2085 & -761 \\
\hline & Brandenburg & 281 Senftenberg/Calau/Spremberg & SPD & $\mathrm{CDU}$ & 1461 & 2 & 880 & 3122 & 3699 \\
\hline & Sachsen-Anhalt & 283 Altmark & SPD & $\mathrm{CDU}$ & 988 & -1 & 777 & 2870 & 3083 \\
\hline & Sachsen-Anhalt & 290 Bernburg/Aschersleben/Quedlinburg & CDU & SPD & 2762 & -7 & 798 & 3185 & 361 \\
\hline & Sachsen-Anhalt & 291 Halle/Altstadt & SPD & $\mathrm{CDU}$ & 192 & -6 & 306 & 1392 & 1290 \\
\hline \multirow[t]{8}{*}{1998} & Mecklenburg-V. & 266 Rostock/Land/Ribnitz/... & SPD & $\mathrm{CDU}$ & 408 & -60 & 3578 & 2147 & -903 \\
\hline & Mecklenburg-V. & 270 Neustrelitz/Strasburg/Pasewalk/... & SPD & $\mathrm{CDU}$ & 13 & 38 & 3330 & 2235 & -1158 \\
\hline & Sachsen-Anhalt & 288 Wittenberg/Gräfenhainichen/... & SPD & $\mathrm{CDU}$ & 4406 & -250 & 4983 & 2622 & 2545 \\
\hline & Sachsen & 308 Delitzsch/Eilenburg/Torgau/Wurzen & SPD & $\mathrm{CDU}$ & 3191 & -135 & 4175 & 2354 & 1640 \\
\hline & Sachsen & 322 Glauchau/Rochlitz/... & SPD & $\mathrm{CDU}$ & 1264 & -30 & 4679 & 2936 & -419 \\
\hline & Sachsen & 323 Chemnitz I & SPD & $\mathrm{CDU}$ & 896 & -103 & 1604 & 752 & 250 \\
\hline & Sachsen & 324 Chemnitz II/Chemnitz-Land & SPD & $\mathrm{CDU}$ & 2257 & -55 & 3167 & 1895 & 1095 \\
\hline & Sachsen & 327 Zwickau/Werdau & $\mathrm{CDU}$ & SPD & 2343 & 19 & 3280 & 2153 & 3508 \\
\hline \multirow[t]{4}{*}{2002} & Mecklenburg-V. & Greifswald/Demmin/Ostvorpommern & $\mathrm{CDU}$ & SPD & 1136 & -38 & 5623 & 2605 & 4078 \\
\hline & Sachsen-Anhalt & Burgenland & SPD & CDU & 1464 & 143 & 6483 & 2654 & -2651 \\
\hline & Sachsen & Chemnitzer Land/Stollberg & $\mathrm{CDU}$ & SPD & 5776 & -3 & 5960 & 2687 & 9043 \\
\hline & Sachsen & Vogtland/Plauen & $\mathrm{CDU}$ & SPD & 1344 & 32 & 6266 & 2760 & 4914 \\
\hline 2005 & Mecklenburg-V. & Bad Doberan/Güstrow/Müritz & SPD & CDU & 2183 & -30 & 2714 & 2094 & 1623 \\
\hline \multirow[t]{2}{*}{2009} & Mecklenburg-V. & Neubrandenburg/Mecklenburg-Strelitz/... & $\mathrm{CDU}$ & $\begin{array}{l}\text { DIE } \\
\text { LINKE }\end{array}$ & 251 & -847 & -1756 & 312 & 536 \\
\hline & Brandenburg & Potsdam/Potsdam-Mittelmark II/... & SPD & $\begin{array}{l}\text { DIE } \\
\text { LINKE }\end{array}$ & 205 & 227 & 67 & -803 & 647 \\
\hline
\end{tabular}

${ }^{a}$ Abgebildet sind Wahlkreise in denen mindestens ein prognostizierter strategischer Stimmentransfer größer ist als der Stimmenvorsprung des Siegers vor dem zweitplatzierten Kandidaten im Wahlkreis

'Pfeile zeigen Richtung strategischen Wählens an z.B. von CDU zu SPD. Negative Werte implizieren strategisches Wählen in die umgekehrte Richtung. Nach 2007 steht PDS stellvertretend für DIE LINKE.

'Stimmenvorsprung des Siegers ohne strategisches Wählen 


\section{Fazit}

Das Phänomen des strategischen Wählens erfreut sich in der Fachwelt seit jeher großen Interesses. Ein wesentlicher Grund hierfür liegt in der Einsicht, dass eine vergleichsweise geringe Zahl von Wählern in der Lage ist, den Ausgang der Wahl zu bestimmen, indem sie sich bewusst gegen ihre präferierte Partei entscheidet. In diesem Beitrag wurde daher die Bedeutung strategischen Erststimmenwählens bei deutschen Bundestagswahlen untersucht. Eine Analyse der offiziellen Ergebnisse aller Wahlkreise in Ostdeutschland für fünf Bundestagswahlen ergab, dass Wähler der strategischen Situation im Wahlkreis Beachtung schenken und ihr Stimmverhalten entsprechend anpassen; d.h. Wähler, deren bevorzugte Partei schlechte Chancen auf den Wahlsieg hatte, wählten häufig das kleinere Übel unter den beiden aussichtsreichsten Parteien. Am meisten profitierten davon 2009 die CDU und DIE LINKE, 1994 die CDU und in den anderen Wahlen die SPD. Am häufigsten Stimmen verloren hat insgesamt die PDS bzw. DIE LINKE. Der Anteil strategischer Wähler im Wahlkreis wurde je nach Wahlkreis und Bundestagswahl auf bis zu $9 \%$ geschätzt; der durchschnittliche Anteil lag je nach Wahl zwischen 2 und $5 \%$. Trotz dieses eher geringen Ausmaßes konnte gezeigt werden, dass strategisches Wählen in der Vergangenheit die Sitzverteilung im Bundestag beeinflusst hat.

Insgesamt waren fünf Direktmandate durch strategisches Wählen betroffen: Die CDU erhielt 1994 in Mecklenburg-Vorpommern durch strategische Stimmen von PDS-Anhängern ein zusätzliches Überhangmandat. Ebenfalls durch strategische Stimmen von PDSAnhängern gewann in demselben Bundesland die SPD 1998 zwei Überhangmandate und die CDU verfehlte aufgrund strategischer Stimmen von PDS-Anhängern zugunsten der SPD den Gewinn eines Überhangmandats in Sachsen. In Sachsen-Anhalt gewann die SPD bei der Bundestagswahl 2002 wiederum durch Stimmen von PDS-Anhängern ein zusätzliches Überhangmandat.

In Bezug auf die im zweiten Abschnitt angesprochene Wahl Helmut Kohls zum Bundeskanzler legen die hier gewonnenen Ergebnisse den Schluss nahe, dass die Kanzlermehrheit 1994 zwar nicht von den strategischen Stimmen der PDS-Anhänger abhing: Lediglich ein CDU-Überhangmandat wäre ohne deren Stimmen im Wahlkreis verloren gegangen - dennoch war der Ausgang knapp. Insgesamt zeigen die Ergebnisse, dass der Einfluss strategischen Wählens auf die Sitzverteilung im Bundestag alles andere als hypothetisch ist. Kurzfristig auf den Ausgang der Wahl hin orientierte Wähler können durchaus den Ausschlag geben. Es ist wichtig, dass Beobachter und Beteiligte dieses Potenzial erkennen und in ihre Beurteilungen des Wahlverhaltens bei deutschen Bundestagswahlen einfließen lassen.

Die vorliegende Studie ist die bislang erste, die einen Einfluss strategischen Wählens auf den Wahlausgang in einzelnen Wahlkreisen nachweisen kann. Da das Augenmerk der Studie allein auf dem Kontext ostdeutscher Wahlkreise liegt, stellt sich die Frage, welche Rolle strategisches Wählen in anderen Ländern mit reinen Mehrheitswahlsystemen spielt. Wie viele Wahlkreise werden etwa in britischen Unterhauswahlen durch kurzfristige strategische Wählerwanderungen entschieden? Angesichts der Schwierigkeiten bei der Bestimmung der Parteianhängerschaften im Wahlkreis lässt sich diese Frage bisher noch nicht beantworten. Sollte es in Zukunft gelingen, die Parteianhängerschaften im Wahlkreis präzise zu ermitteln, so ließe sich mit dem hier vorgestellten Modell auch 
der Einfluss strategischen Wählens in reinen Mehrheitswahlsystemen bestimmen. Da die Sitzverteilung in diesen Systemen - anders als in Deutschland - einzig von den Wahlausgängen in den lokalen Wahlkreisen abhängt, wäre zu vermuten, dass strategisches Wählen dort einen noch größeren Einfluss auf die Kräfteverhältnisse im Parlament haben sollte. Es wäre daher eine äußerst spannende Aufgabe, dieser Frage weiter nachzugehen.

\section{Anhang}

Ungleichung (2) lässt sich aus Ungleichung (1) wie folgt herleiten:

$$
\begin{aligned}
p_{12}\left(u_{1}-u_{2}\right)+p_{13}\left(u_{1}-u_{3}\right) & \geq p_{12}\left(u_{2}-u_{1}\right)+p_{23}\left(u_{2}-u_{3}\right) \\
p_{12}\left(u_{1}-u_{2}\right)+p_{13}\left(u_{1}-u_{3}\right) & \geq-p_{12}\left(u_{1}-u_{2}\right)+p_{23}\left(u_{2}-u_{3}\right) \\
2 p_{12}\left(u_{1}-u_{2}\right)+p_{13}\left(u_{1}-u_{3}\right) & \geq p_{23}\left(u_{2}-u_{3}\right) \\
2 p_{12}\left(u_{1}-u_{2}+u_{3}-u_{3}\right)+p_{13}\left(u_{1}-u_{3}\right) & \geq p_{23}\left(u_{2}-u_{3}\right) \\
2 p_{12}\left(u_{1}-u_{3}\right)-2 p_{12}\left(u_{2}-u_{3}\right)+p_{13}\left(u_{1}-u_{3}\right) & \geq p_{23}\left(u_{2}-u_{3}\right) \\
2 p_{12}\left(u_{1}-u_{3}\right)+p_{13}\left(u_{1}-u_{3}\right) & \geq 2 p_{12}\left(u_{2}-u_{3}\right)+p_{23}\left(u_{2}-u_{3}\right) \\
\left(2 p_{12}+p_{13}\right)\left(u_{1}-u_{3}\right) & \geq\left(2 p_{12}+p_{23}\right)\left(u_{2}-u_{3}\right) \\
\frac{u_{1}-u_{3}}{u_{2}-u_{3}} & \geq \frac{2 p_{12}+p_{23}}{2 p_{12}+p_{13}}
\end{aligned}
$$

\section{Literatur}

Alvarez, Michael R., und Jonathan Nagler. 2000. A new approach for modelling strategic voting in multiparty elections. British Journal of Political Science 30:57-75.

Alvarez, Michael R., Frederick J. Boehmke, und Jonathan Nagler. 2006. Strategic voting in British elections. Electoral Studies 25:1-19.

Bawn, Kathleen. 1999. Voter responses to electoral complexity: Ticket splitting, rational voters and representation in the Federal Republic of Germany. British Journal of Political Science 29:487-505.

Behnke, Joachim. 2003. Ein integrales Modell der Ursachen von Überhangmandaten. Politische Vierteljahresschrift 44:41-65.

Behnke, Joachim. 2007. The strange phenomenon of surplus seats in the German electoral system. German Politics 16:496-517.

Black, Jerome H. 1978. The multicandidate calculus of voting: Application to Canadian federal elections. American Journal of Political Science 22:609-638.

Blais, André, und Richard Nadeau. 1996. Measuring strategic voting: A two-step procedure. Electoral Studies 15:39-52.

Blais, André, Richard Nadeau, Elisabeth Gidengil, und Neil Nevitte. 2001. Measuring strategic voting in multiparty elections. Electoral Studies 20:343-352.

Blais, André, Robert Young, und Martin Turcotte. 2005. Direct or indirect? Assessing two approaches to the measurement of strategic voting. Electoral Studies 24:163-176.

Cain, Bruce E. 1978. Strategic voting in Britain. American Journal of Political Science 22:639-655.

Cox, Gary W. 1997. Making votes count: Strategic coordination in the world's electoral systems. Cambridge: Cambridge University Press. 
Fey, Mark. 1997. Stability and coordination in Duverger's Law: A formal model of preelection polls and strategic voting. American Political Science Review 91:135-147.

Fieldhouse, Edward A., Charles J. Pattie, und Ronald J. Johnston. 1996. Tactical voting and party constituency campaigning at the 1992 general election in England. British Journal of Political Science 26:403-439.

Fisher, Stephen D. 2004. Definition and measurement of tactical voting: The role of rational choice. British Journal of Political Science 34:125-166.

Galbraith, John W., und Nicol C. Rae. 1989. A test of the importance of tactical voting: Great Britain, 1987. British Journal of Political Science 19:126-136.

Greene, William H. 2003. Econometric analysis. Upper Saddle River: Prentice Hall.

Gschwend, Thomas. 2007. Ticket-splitting and strategic voting under mixed electoral rules: Evidence from Germany. European Journal of Political Research 46:1-23.

Gschwend, Thomas, Ronald J. Johnston, und Charles Pattie. 2003. Split-ticket patterns in mixedmember proportional election systems: Estimates and analyses of their spatial variation at the German federal election, 1998. British Journal of Political Science 33:109-127.

Heath, Anthony, Roger Jowell, John Curtice, Geoffrey Evans, Julia Field, und Sharon Witherspoon. 1991. Understanding political change. The British voter 1964-1987. Oxford: Pergamon.

Herrmann, Michael. 2008. Estimating uncertainty in strategic voting. Konferenzpapier, European Public Choice Society.

Herrmann, Michael, und Franz U. Pappi. 2008. Strategic voting in German constituencies. Electoral Studies 27:228-244.

Hoffman, Dale T. 1982. A model for strategic voting. SIAM Journal on Applied Mathematics 42:751-761.

Johnston, Ronald J., und Charles Pattie. 1991. Tactical voting in Great Britain 1983 and 1987: An alternative approach. British Journal of Political Science 21:95-128.

Karp, Jeffrey A., Jack Vowles, Susan A. Banducci, und Todd Donovan. 2002. Strategic voting, party activity, and candidate effects: Testing explanations for split voting in New Zealand's new mixed system. Electoral Studies 21:1-22.

Lijphart, Arend. 1994. Electoral systems and party systems. A study of twenty-seven democracies, 1945-1990. Oxford: Oxford University Press.

McKelvey, Richard D., und Peter C. Ordeshook. 1972. A general theory of the calculus of voting. In Mathematical applications in political science, Hrsg. James F. Herndon und Joseph L. Bernd, 32-78. Charlottesville: University Press of Virginia.

Myatt, David P. 2007. On the theory of strategic voting. Review of Economic Studies 74:255-281.

Myatt, David P., und Stephen D. Fisher. 2002. Everything is uncertain and uncertainty is everything. Department of Economics Discussion Paper 115. University of Oxford.

Myerson, Roger B., und Robert J. Weber. 1993. A theory of voting equilibria. American Political Science Review 87:102-114.

Niemi, Richard G., Guy Whitten, und Mark N. Franklin. 1992. Constituency characteristics, individual characteristics and tactical voting in the 1987 British general election. British Journal of Political Science 22:229-254.

Ordeshook, Peter C., und Langche Zeng. 1997. Rational voters and strategic voting: Evidence from the 1968, 1980 and 1992 elections. Journal of Theoretical Politics 9:167-187.

Palfrey, Thomas R. 1989. A mathematical proof of Duverger's Law. In Models of strategic choice in politics, Hrsg. Peter C. Ordeshook, 69-91. Ann Arbor: University of Michigan Press.

Pappi, Franz U., und Paul W. Thurner. 2002. Electoral behavior in a two-vote system: Incentives for ticket-splitting in German Bundestag elections. European Journal of Political Research 41:207-232.

Rallings, Colin, und Michael Thrasher. 2007. British electoral facts 1832-2006. Aldershot: Ashgate. 
Reed, Steven R. 1990. Structure and behaviour: Extending Duverger's Law to the Japanese case. British Journal of Political Science 20:335-356.

Riker, William H., und Peter C. Ordeshook. 1968. A theory of the calculus of voting. American Political Science Review 62:25-42.

Schoen, Harald. 1998. Stimmensplitting bei Bundestagswahlen: Eine Form taktischer Wahlentscheidung? Zeitschrift für Parlamentsfragen 29:223-244.

Shikano, Susumu, Michael Herrmann, und Paul W. Thurner. 2009. Strategic voting under proportional representation: Threshold insurance in German elections. West European Politics 32:630-652. 